\section{Developing form}

On Development: The Biology of Form. By J. T. Bonner. Pp. 282. (Harvard University Press, Cambridge, 1974.) $\$ 10.00$.

Do not expect to find here a fashionable account of developmental biology in terms of molecular mechanisms controlling transcription and translation, or specificity of cell surfaces, or automata theory. For this book attempts to deal with developmental biology in a very broad manner, by considering not only mechanisms in a general way but by considering also the problems of evolution and population biology.

Bonner attempts to do this by emphasising the importance of life cycles, which he believes throws new light on basic problems. He views, for example, biological reproduction in terms of reproduction of the life cycle and this theme is expanded in the first part of the book to discuss size increase in evolution, senescence, variation, the origin of life multicellularity. An essential feature of a life cycle is not only to reproduce a new cycle, but to get rid of the old one, and he thus argues for programmed senescence. (No mention is made, however, of Hayflick's studies on the limited life span of somatic cells.) Some attention is given to looking at life cycles as having points of maximum and minimum size, and size increase during evolution is discussed in some detail from the point of view of selective advantage. There is also a quite long digression on the origin of social organisms. His aim is to say something about the larger significance of development, such as why development occurs, how it evolved, and its relationship to all other aspects of life. Interesting though this biology may be, I have not found viewing it in terms of life cycles very helpful, nor do I consider it to be an advance over the distinction between genotype and phenotype. By looking at the selective advantage of the phenotype he misses the really interesting questions on the relationship between evolution and development. What we want to know is what constraints and integrations development provides when the genotype changes. How, for example, do new cell types arise? What is the selective advantage as they are evolving? One can ask the same question of the evolution of structural innovations such as the wing. Again, what sort of changes in genotype are required in the alteration in the pattern of muscular activity. Must one alter genes controlling both nerve and muscle, or will developmental mechanisms provide the required integration? Could one by appropriate selection evolve a bat-like wing for man? Dobzhansky has said no, and thus man has no hope of becoming an angel, but I do not believe current theory permits a decision. None of these problems is even recognised in this book.

The second part of the book is concerned with the mechanisms of development and covers topics such as synthesis of substances, timing, localisation of substances and control of pattern, and is rather disappointing. Part of the difficulty is that Bonner seems much more at home with protozoa, fungi, slime moulds and plants. This may account for the fact that most of the examples are drawn from such fields and not only are they less familiar, but in general, much less is known about them. This also accounts for the rather cursory treatment of topics such as microtubules and microfilaments and cell movement in general. One could argue that for animal development this was an area of major importance in which rapid advances are being made. Other areas such as clonal analysis, which are being applied with remarkable success to insect development are not even mentioned.

The book is characterised in places by a regrettable lack of rigour. For example, the brief analogy drawn between the brain and the structure of the genome is very superficial, in that it would be as true of a juke box. Again, a principle of convergence is invoked to suggest that particular biological functions in development can be fulfilled in a variety of ways; but no attention is given to a principle of conservation which would suggest that once embryos have found a good way of doing things they will continue to use it. In fact, one of the major failures of the book is to bring out common features and general principles in development. Yet again, the author suggests that it is a distinct selective advantage for the grouping of developmental events into superunits of gene action, but very little evidence is available to substantiate such a view: the interconnectedness of genes remains a major problem and is not seriously analysed here. Later, he suggests that there are not enough genes to account for all individual neurones, completely ignoring the tremendous combinatorial possibilities of gene networks which could easily and uniquely characterise each neurone with a relatively small number of genes.

In spite of these criticisms, the author draws on a wide range of biological examples and his ideas are often provocative and original. By raising problems ranging from the development of the social behaviour of ants to the form of mushrooms he presents to developmental biologists problems and ideas that rarely cross their minds.

Lewis Wolpert
New from Addison-Wesley Advanced Book Program

M. A. PRESTON and R. K. BHADURI

McMaster University

Structure of the Nucleus

This graduate-level text and reference work is the direct successor to Professor Preston's "Physics of the Nucleus" brought about by substantial developments in nuclear physics since 1962. Nuclear structure is emphasized throughout the three parts: Fundamental Properties of Nuclei; Nuclear Models; Alpha Disintegration and Fission of Nuclei.

Spring 1975 , about 700 pp., illus. hardbd. ISBN 0-201-05976-2,

approx. $£ 13.75$

paperbd. ISBN 0-201-05977-0,

approx. $£ 8.25$

MURRAY SARGENT III,

MARLAN O. SCULLY, and

WILLIS E. LAMB, JR.

University of Arizona

\section{Laser Physics}

This volume treats the interaction of radiation with matter paying particular attention to the laser. Because existing laser media are intrinsically quantum mechanical and are most easily studied with the quantum theory, this book not only uses quantum mechanics but enlivens the reader's understanding of the subject as well. As a result the material constitutes a viable, applied alternative to the usual undergraduate courses on quantum mechanics. The reader will obtain from this presentation not only an increased understanding of the laser, but a more profound comprehension of many-system physical phenomena as well. 1974, xxviii, 432 pp., illus.

hardbd. ISBN 0-201-06718-8, £12.10 paperbd. ISBN 0-201-06719-6, £7.45

\section{PHYSICS OF QUANTUM}

ELECTRONICS

Based on Lectures of Summer Schools

Vol. 1: High Energy Lasers

and Their Applications

Edited by Stephen Jacobs,

Murray Sargent III, and

Marlan O. Scully

1974, xii, 411 pp., ISBN 0-201-05680-X, f9.65

Vol. 2

Laser Applications to Optics and Spectroscopy

Edited by Stephen F. Jacobs,

Murray Sargent III,

James F. Scott, and

Marlan O. Scully

1975, xii, 510 pp., ISBN 0-201-05682-8, $£ 12.95$

Addison-Wesley Publishers, Ltd.

West End House Advanced Book Program 11 Hills Place Reading, Mass. 01867 London

U.S.A. 